\title{
A rare penetrant TIMP3 mutation confers relatively late onset choroidal neovascularisation which can mimic age-related macular degeneration
}

${ }^{1}$ Clinical Neurosciences Research Group, Clinical and Experimental Sciences, Faculty of Medicine, University of

Southampton,

Southampton, UK

${ }^{2}$ Eye Unit, University Southampton NHS Trust, Southampton, UK

${ }^{3}$ Centre for Biological Science, Faculty of Natural and Environmental Sciences, University of Southampton,

Southampton, UK

Correspondence: A Lotery, Division of Neurosciences, University of Southampton UK, Faculty of Medicine, University of Southampton, South Lab and Path Block, Mailpoint 806, Level D, Southampton, Hampshire SO16 6YD, UK Tel: +44 238079 5049; Fax: +44 2380794542 . E-mail: a.j.lotery@ soton.ac.uk

\begin{abstract}
Purpose To perform a genotype-phenotype correlation for three patients heterozygous for a missense mutation in the tissue inhibitor of metalloproteinase 3 (TIMP3) gene.

Methods Retrospective, observational case series. The medical records and photographs were reviewed for three patients diagnosed at the time with neovascular age-related macular degeneration (AMD). All were later found to carry a predicted C113G mutation in the TIMP3 gene, other known mutations in which are associated with Sorsby's fundus dystrophy. Results All three patients developed drusen and bilateral choroidal neovascularisation with subsequent disciform scarring and atrophy. Visual acuity rapidly deteriorated to $<6 / 60$ in both eyes. The age of onset varied from 56 to 64 years and the interval to contralateral eye involvement varied from 4 to 6 years. Two of the three patients had a family history of AMD. All three patients were heterozygous for the C113G nucleotide change, resulting in a Ser38Cys change at the $\mathbf{N}$ terminus of the TIMP3 protein. Conclusion This case series suggests the C113G TIMP3 variant may represent a novel highly penetrant mutation causing choroidal neovascularisation of relatively late onset for Sorsby's fundus dystrophy, mimicking early onset AMD.
\end{abstract}

Eye (2016) 30, 488-491; doi:10.1038/eye.2015.204; published online 23 October 2015

\section{Introduction}

Age-related macular degeneration (AMD) is the commonest cause of blindness in the developed
A Warwick ${ }^{1,2}$, J Gibson ${ }^{3}$, R Sood ${ }^{3}$ and A Lotery L $^{1,2}$ world. ${ }^{1}$ Genetic predisposition is thought to play a role and to date 19 genetic loci have been associated at a genome-wide level with an increased risk of developing AMD. ${ }^{2}$

Sorsby's fundus dystrophy (SFD) is a rare autosomal dominant fundus dystrophy caused by mutations in the tissue inhibitor of metalloproteinase 3 (TIMP3) gene. ${ }^{3}$ It shares similar clinical features with AMD, including choroidal neovascularisation (CNV), although the age of onset is typically younger in the fourth decade of life.

We report the clinical phenotypes of three patients diagnosed at Southampton Eye Unit with bilateral neovascular AMD before the availability of anti-vascular endothelial growth factor (VEGF) therapies. All three were later found to be heterozygous for a predicted mutation, C113G, in the TIMP3 gene. These genotypes were identified through a collaboration with the International AMD Genomics Consortium (IAMDGC). ${ }^{4}$

\section{Case reports}

\section{Case 1}

A 56-year-old man presented with recent deterioration of vision in his left eye. Visual acuity was 6/9 in the right eye and 3/60 in the left eye. Examination revealed haemorrhage and subretinal fluid at the left macula. He had a family history of 'macular degeneration'.

He then returned to the eye unit 4 years later with 'advanced macular degeneration' in the right eye. Visual acuity was now 2/60 in the right eye and hand movements in the left eye due to disciform scarring. 

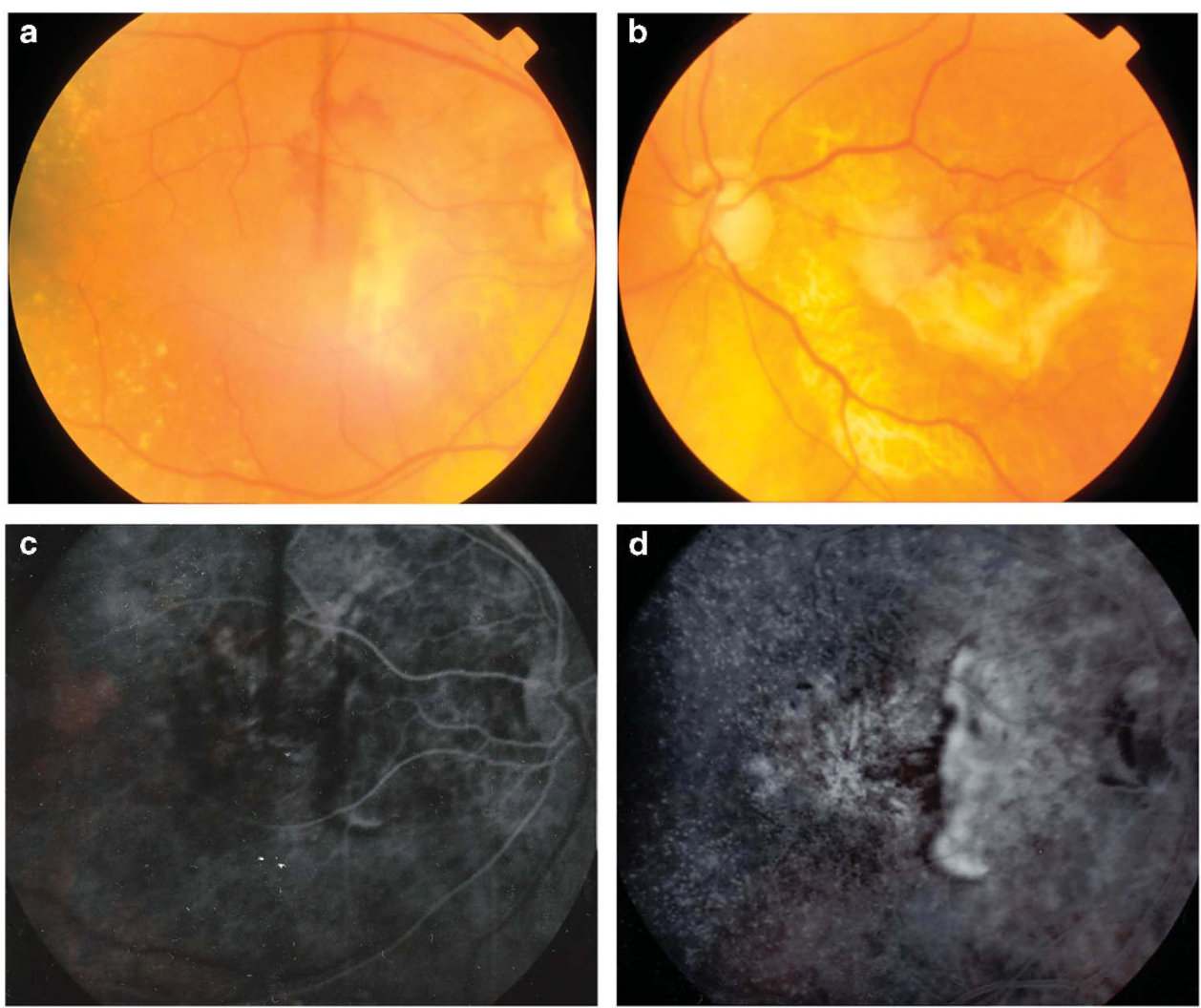

Figure 1 Fundus photographs of patient 2 taken at the onset of right eye visual symptoms. Haemorrhage at the right macula (a), disciform scarring in the left eye (b) and bilateral drusen are shown. Early (c) and late (d) fluorescein angiography in the right eye revealed peripapillary choroidal neovascularisation.

\section{Case 2}

A 64-year old man presented with sudden-onset reduced vision in his left eye. Visual acuity was $6 / 6$ in the right eye and counting fingers in the left. Fluorescein angiography revealed a left subfoveal classic $\mathrm{CNV}$. Both his grandmother and uncle had also been diagnosed with AMD.

Five years later his vision had decreased to 6/36 in the right eye. Fundus examination showed bilateral drusen with macular haemorrhage in the right eye and a left disciform scar (Figure 1a and b). Fluorescein angiography revealed a peripapillary CNV (Figure 1c and d). This was treated with radiotherapy, however by the following year his vision had decreased further to $6 / 60$.

\section{Case 3}

A 61-year old woman developed choroidal neovascularisation in her left eye, reducing her vision to hand movements. She had no family history of AMD. Six years later she developed symptoms in her right eye.

Examination revealed bilateral drusen, disciform scarring in the left eye and haemorrhage at the right macula (Figure 2a and b). Indocyanine green angiography findings suggested right polypoidal choroidopathy
(Figure 2c and d). Despite treatment with photodynamic therapy her visual acuity deteriorated from $6 / 36$ to hand movements a year later.

\section{Discussion}

TIMP3 is a $24 \mathrm{kD}$ protein that inhibits matrix metalloproteinases. It is secreted by the retinal pigment epithelium (RPE) and deposits in Bruch's membrane where it regulates extracellular matrix turnover, ${ }^{5}$ inhibits angiogenesis ${ }^{6}$ and regulates inflammation. ${ }^{7}$ Eleven distinct mutations to date have been associated with Sorsby's fundus dystrophy. All of these create unpaired cysteine residues in the C-terminal domain of the TIMP3 protein. ${ }^{8}$ This results in the formation of dimers which accumulate in Bruch's membrane, compromising extracellular matrix turnover. ${ }^{9}$ RPE function is subsequently affected, leading to RPE atrophy and CNV.

The three patients described, diagnosed at the time with bilateral 'neovascular AMD', were found to be heterozygous for a predicted mutation, C113G, causing a Ser38Cys change and unpaired cysteine residue in the $\mathrm{N}$ terminus of TIMP3. The only other published report of this mutation describes two patients with strong family histories of early onset 

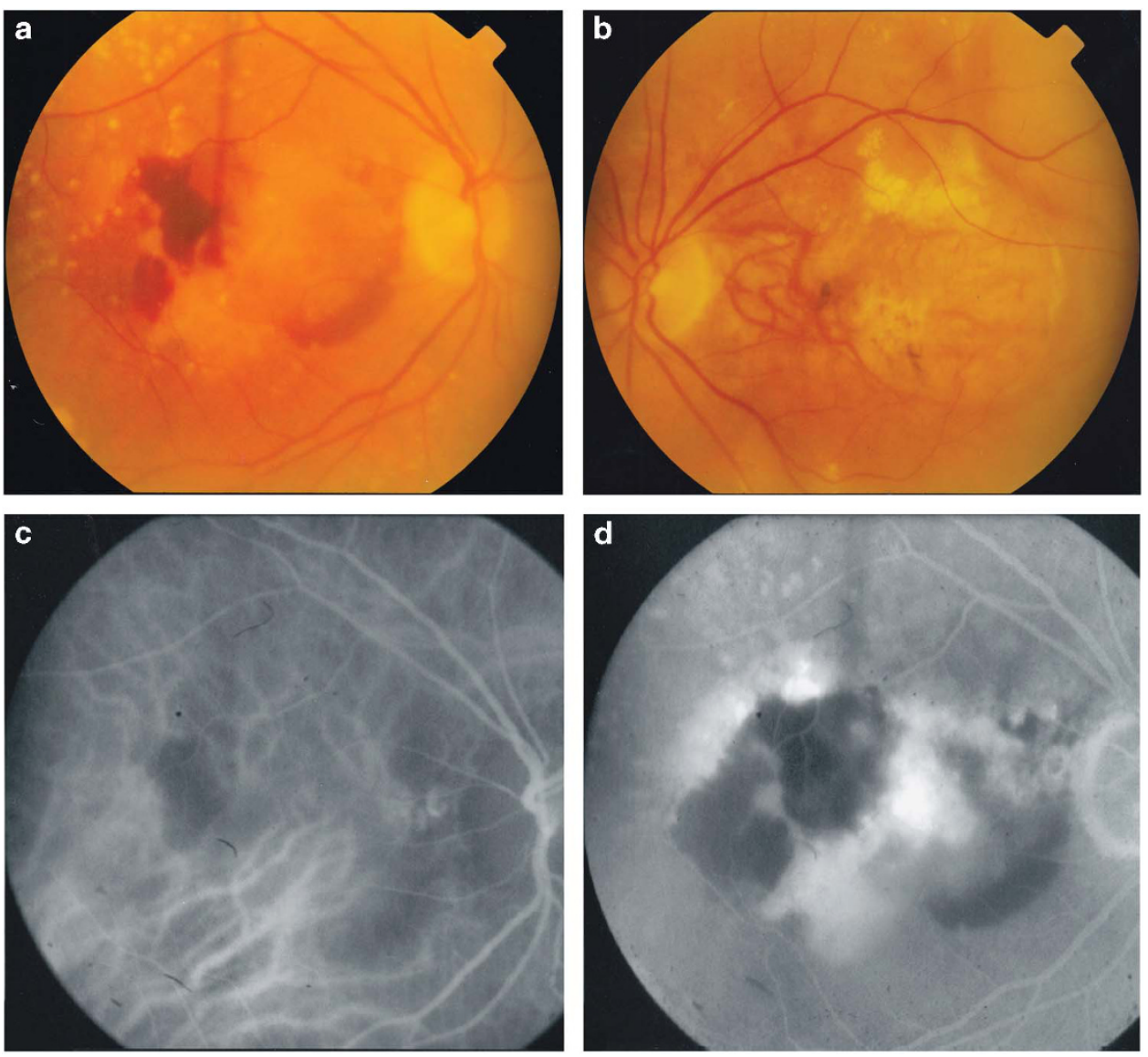

Figure 2 Fundus photographs of patient 3 taken at the onset of right eye visual symptoms, showing bilateral drusen, right eye macular haemorrhage (a) and a left disciform scar (b). Early (c) and late (d) indocyanine green angiography suggested polypoidal choroidopathy temporal to the right optic disc.

AMD who developed bilateral recurrent choroidal neovascularisation in their forties. ${ }^{8}$ By contrast, our three patients developed visual symptoms between 56 and 64 years, and one patient had no family history of AMD.

Recently presented work from the IAMDGC identified 28 heterozygotes for rare TIMP3 mutations and variants within 16144 advanced AMD cases versus one heterozygote among $>20000$ controls, which includes our three cases. ${ }^{4}$ The majority of these carried the C113G mutation and the average age of onset for macular disease was 65 years. This was significantly earlier than for non-carriers (77 years old, $P<0.000001$ ) but older than is typical for SFD.

We therefore conclude that the C113G TIMP3 mutation phenotype may mimic early onset neovascular AMD. Our report represents the first genotype-phenotype correlation of this mutation emerging from the IAMDGC. Cascade genetic testing for this rare highly penetrant allele in the relatives of patients with CNV diagnosed as early onset AMD may identify asymptomatic carriers who require close monitoring for future development of macular disease. This would aid prompt initiation of antiVEGF therapy to prevent sight loss. ${ }^{10}$

\section{Summary}

What was known before

- A number of genetic variants have been associated with increased risk of developing age-related macular degeneration (AMD).

- Sorsby's fundus dystrophy (SFD) is a rare autosomal dominant fundus dystrophy caused by mutations in the TIMP3 gene.

- SFD shares certain clinical features with AMD, however the age of onset is typically younger.

\section{What this study adds}

- We report a genotype-phenotype correlation for three patients diagnosed at the time with early onset neovascular AMD, who were later found to carry a 'predicted' mutation in the TIMP3 gene (C113G).

- Our report represents the first genotype-phenotype correlation of this mutation emerging from the International AMD Genomics Consortium.

- We conclude that the C113G TIMP3 mutation may represent a novel highly penetrant mutation causing choroidal neovascularisation of relatively late onset for Sorsby's fundus dystrophy, mimicking early onset AMD. Cascade genetic testing in the relatives of affected patient may identify asymptomatic carriers who require close monitoring for future development of macular disease. 


\section{Conflict of interest}

The authors declare no conflict of interest.

\section{Acknowledgements}

The International AMD Genomics Consortium who assisted with genotyping. The Wellcome Trust Clinical Research Facility Southampton who assisted with DNA collection. Ms Helen Griffiths and Ms Angela Cree for DNA extraction. Funding from the Gift of Sight Appeal and the Rosetree Trust.

\section{References}

1 Klein R, Peto T, Bird A, Vannewkirk MR. The epidemiology of age-related macular degeneration. Am J Ophthalmol 2004; 137: 486-495.

2 Fritsche LG, Chen W, Schu M, Yaspan BL, Yu Y, Thorleifsson $\mathrm{G}$ et al. Seven new loci associated with age-related macular degeneration. Nat Genet 2013; 45: 433-2.

3 Weber BH, Vogt G, Wolz W, Ives EJ, Ewing CC. Sorsby's fundus dystrophy is genetically linked to chromosome 22q13-qter. Nat Genet 1994; 7: 158-161.
4 Fritsche LG. International AMD Genomics Consortium. The role of rare TIMP3 mutations in Age-Related Macular Degeneration. The American Society of Human Genetics, October 21, 2014, San Diego, CA, USA, 2014.

5 Della NG, Campochiaro PA, Zack DJ. Localization of TIMP-3 mRNA expression to the retinal pigment epithelium. Invest Ophthalmol Vis Sci 1996; 37: 1921-1924.

6 Qi JH, Ebrahem Q, Moore N, Murphy G, Claesson-Welsh L, Bond $\mathrm{M}$ et al. A novel function for tissue inhibitor of metalloproteinases-3 (TIMP3): inhibition of angiogenesis by blockage of VEGF binding to VEGF receptor-2. Nat Med 2003; 9: 407-415.

7 Mohammed FF, Smookler DS, Taylor SE, Fingleton B, Kassiri Z, Sanchez $\mathrm{OH}$ et al. Abnormal TNF activity in Timp3-/- mice leads to chronic hepatic inflammation and failure of liver regeneration. Nat Genet 2004; 36: 969-977.

8 Schoenberger SD, Agarwal A. A novel mutation at the $\mathrm{N}$-terminal domain of the TIMP3 gene in Sorsby fundus dystrophy. Retina 2013; 33: 429-435.

9 Jacobson SG, Cideciyan AV, Bennett J, Kingsley RM, Sheffield VC, Stone EM. Novel mutation in the TIMP3 gene causes Sorsby fundus dystrophy. Arch Ophthalmol 2002; 120: 376-379.

10 Gemenetzi MK, Luff AJ, Lotery AJ. Successful treatment of choroidal neovascularization secondary to sorsby fundus dystrophy with intravitreal bevacizumab. Retin Cases Brief Rep 2011; 5: 132-135. 